\title{
Deficient learning and memory in early and middle phases of multiple sclerosis
}

\author{
IGOR GRANT, ${ }^{*}$ W IAN MCDONALD,$\dagger$ MICHAEL R TRIMBLE, $\dagger$ EILEEN SMITH, \\ ROBERT REED*
}

From the Psychiatry Service, San Diego V.A. Medical Center and Department of Psychiatry, University of California at San Diego School of Medicine, La Jolla, California, USA, ${ }^{*}$ the Institute of Neurology and National Hospitals, Queen Square and Maida Vale, $†$ and the National Hospitals (Maida Vale), $\ddagger$ London, UK

SUMMARY Forty-three patients with multiple sclerosis showed disturbances in short-term memory, learning, and delayed recall which were associated with years of active disease (average was 4.5 years), age, presence of flareup, but not steroid/ACTH treatment. Unrecognised memory loss might be prevalent early in the natural history of multiple sclerosis and deserves neuropsychological assessment.

Cognitive decline commonly occurs in the later stages of multiple sclerosis. ${ }^{1}$ In the past three decades some of the qualitative and quantitative features of cognitive change have been delineated through neuropsychological inquiry. Many investigations of intelligence in multiple sclerosis have concluded that patients with demyelination show disproportionate drops in peformance IQ while maintaining relatively good verbal skills. ${ }^{2}$ More comprehensive neuropsychological studies, utilising procedures such as the Halstead-Reitan Battery revealed patients with multiple sclerosis to have marked motor and perceptual-motor integrative deficits, generally intact verbal-language skills, and variable decrements in abstracting ability. ${ }^{3-10}$

A century has passed since Charcot first observed "enfeeblement of memory" in his patients" and Gowers wrote of their "failure of memory". ${ }^{12}$ Despite this, the neuropsychological research reviewed above has been singularly lacking in systematic assessment of memory. Surridge ${ }^{13}$ noted from structured psychiatric examination that approximately two-thirds of his patients showed intellectual decline, with amnesia being a central problem. Jambor's ${ }^{14}$ related psychometric study found deficits in sentence learning and delayed recall of nonverbal information. These observations

Address for reprint requests: Professor I Grant, Psychiatry Service (116). V.A. Medical Center, San Diego, CA 92161, USA

Received 1 August 1983

Accepted 24 September 1983 were based on a group of patients about half of whom had the disease 6-10 years, and a third, 11 years or longer.

Beatty and Gange ${ }^{15}$ examined a group of patients who were ill for approximately nine years. They found deficiencies in learning word lists and in delayed recall of a short story. Staples and Lincoln ${ }^{16}$ showed that multiple sclerosis patients with severe disability had worse learning and delayed recall both on verbal and nonverbal tasks, when compared to a matched sample of patients with muscular dystrophy.

These few systematic studies appear to confirm clinical observations and patient self-reports of impaired memory in association with established and advanced multiple sclerosis. The purpose of this study has been to expand our understanding of memory pathology in multiple sclerosis by: (1) examining a more complete range of memory processes than has been attempted heretofore; (2) considering whether memory pathology occurs commonly at a relatively early stage in the natural history of multiple sclerosis.

\section{Method}

\section{SUBJECTS}

(a) Multiple Sclerosis Group $(N=43)$

Patients with multiple sclerosis were inpatients and outpatients at the National Hospitals, Queen Square and Maida Vale. Because some of these patients were also selected to be participants in a related study (life events and multiple sclerosis, to be reported separately) which called for enrolling patients in the early phase of their disease, the major- 
ity carried the diagnosis for less than 5 years. The mean number of years of active disease (defined as number of years in which the patient reported at least one week's duration of symptoms) was $4 \cdot 47 \pm 3 \cdot 56$, with a range of 1-15 years. Fifty-two patients were examined initially, but nine were later excluded because they failed to meet the diagnostic criteria of "early probable" or "clinically definite" multiple sclerosis. ${ }^{17}$

The specific reasons for exclusion were possible multiple sclerosis $(N=6)$, uncertain diagnosis $(N=1)$, demyelination confounded by another neurological disorder $(\mathrm{N}=1)$, and establishment after enrollment of another definite diagnosis $(\mathrm{N}=1)$. For the retained patients, the distribution of diagnoses, based on a careful review of all clinical and laboratory data by one of the authors (WIMcD) was: 31 patients with clinically definite multiple sclerosis; one patient with early progressive multiple sclerosis; and 11 patients with probable multiple sclerosis. There were 27 women and 16 men, and their age was $37.8 \pm 10 \cdot 2$ (range 19-56). Twenty-nine were inpatients and 14 were outpatients at the time of neuropsychological testing.

\section{Healthy Comparison Group $(N=28)$}

This sample consisted of normal subjects who were comparable in age, sex distribution, and estimated intelligence to the patient group. There were 17 women and 11 men whose average age was $34 \cdot 8 \pm 10 \cdot 3$ (range 18-54), and whose Vocabulary scale score (from the Wechsler Adult Intelligence Scale, or WAIS) was $12 \cdot 3 \pm 2 \cdot 7$. Their vocabulary performance was comparable to that of the multiple sclerosis patients $(11.9 \pm 2.3 ; \mathrm{F}(1,69)=0.52$, ns), and placed both groups into an average intelligence (est. IQ $=110$ ) bracket.

\section{NEUROPSYCHOLOGICAL TESTS}

\section{WAIS Vocabulary (18)}

This subtest from the Wechsler Adult Intelligence Scale requires subjects to define words whose frequency of occurrence ranges from common to infrequent. Beyond reflecting language skills, performance on Vocabulary is highly correlated with overall intelligence.

\section{TRAIL MAKING TEST (19)}

This component of the Halstead-Reitan Battery has two parts. The simpler (Part $A$ ) requires subjects to use a pencil to connect circled numbers displayed on a page as quickly as they can. Part B displays numbers and letters; using a pencil, subjects must alternate between numbers and letters while maintaining the correct order in the number and letter strings. Time and accuracy are scored. This psychomotor test requires motor speed and steadiness as well as ability to order symbols presented in a visual array.

WECHSLER MEMORY SCALE (WMS) LOGICAL AND VISUAL MEMORY COMPONENTS (20)

\section{Verbal Learning and Delayed Recall}

We selected one of the prose passages from the WMS, read it to our subjects, asked them to repeat the passage verbatim after a 10 second delay, and immediately scored the number of memories produced. For the story, 22 memories was the maximum possible; if a person did not reach at least $\mathbf{1 5}$ memories on the first recollection, we presented the story again and required a second recall. This process was continued for a maximum of five trials if a person did not reach the criterion of 15 before then. Subjects were then asked to recall the prose passage $45 \mathrm{~min}$ later. Other testing occupied the interim. Three scores could be developed from this procedure: an immediate recall score (that is, number of memories at trial 1); a learning score (number of trials to reach criterion); and a delayed recall score (number of memories at $45 \mathrm{~min}$ ).

\section{Visual Learning and Recall}

The Wechsler Memory Scale has three geometric stimulus cards, cards 1 and 2 containing one figure each, and card 3, two figures. Each card was presented to a subject for 10 seconds, and then the subject was required to reproduce each figure 10 seconds later on a piece of paper. The same principles of criterion learning and $45 \mathrm{~min}$ delayed recall were used as for the story above. Immediate, trials to criterion, and delayed recall scores were computed.

BROWN-PETERSON TEST (21-22)

The Brown-Peterson distractor technique is a measure of short-term retention, and has been widely used as a test of short-term memory. Subjects were shown a card containing three consonants (a consonant trigram). There were two experimental manoeuvres which could, theoretically, either facilitate or interfere with short-term retention. To determine whether retention could be facilitated by rehearsal in our patients, they were required sometimes to recollect the trigram immediately after presentation, sometimes after a 2 second delay and other times after a 4 second delay. To determine our patients' sensitivity to "proactive interference" they were sometimes required to recollect the trigram after periods of $3,6,9$ or 18 seconds of counting backwards by threes; other times no counting was required. There were 30 trigrams presented, randomised across the three rehearsal and five interference conditions. Subjects were allowed a rest period of 6 seconds between trials. The specific modification of the BrownPeterson technique is very similar to the one employed by Butters and associates ${ }^{23}$ in their comparison of short-term memory in patients with early and late Huntington's Disease.

\section{Results}

\section{Comparison of multiple sclerosis patients with non- patients}

Table 1 compares neuropsychological performance of multiple sclerosis patients with that of nonpatients. It will be seen that although multiple sclerosis patients were comparable in age and verbal intelligence to their nonpatient counterparts, they performed significantly worse on all other neuropsychological tests. Specifically, they were very significantly impaired on both Trail Making tests. It appears that the psychomotor speed demand of these tests was primarily responsible for the difficulties experienced by patients, since they had about 
Table 1 Learning and memory in multiple sclerosis. Neuropsychological test results

\begin{tabular}{|c|c|c|c|c|}
\hline \multirow[t]{2}{*}{ Tests } & \multicolumn{2}{|l|}{ Groups* } & \multicolumn{2}{|l|}{ Statistics } \\
\hline & $\begin{array}{l}\text { Patients } \\
N=43\end{array}$ & $\begin{array}{l}\text { Nonpatients } \\
N=28\end{array}$ & $F(1,69)$ & $p<$ \\
\hline WAIS Vocabulary & $11.9 \pm 2.3$ & $12 \cdot 3 \pm 2 \cdot 7$ & $1 \cdot 52$ & ns \\
\hline $\begin{array}{l}\text { Trailmaking } \\
\text { Part A (time-second) } \\
\text { Trailmaking } \\
\text { Part B (time-second) }\end{array}$ & $\begin{aligned} 51 \cdot 4 & \pm 25 \cdot 8 \\
105 \cdot 8 & \pm 56 \cdot 3\end{aligned}$ & $\begin{array}{l}27 \cdot 9 \pm 9 \cdot 3 \\
65 \cdot 7 \pm 22 \cdot 8\end{array}$ & $\begin{array}{l}21 \cdot 0 \\
12 \cdot 6\end{array}$ & $\begin{array}{l}0.0001 \\
0.001\end{array}$ \\
\hline $\begin{array}{l}\text { WMS story } \\
\text { Trial } 1 \text { (\# bits) } \\
\text { Trials to criterion } \\
45 \text { minute delay }\end{array}$ & $\begin{array}{r}8.4 \pm 3.4 \\
3.2 \pm 1.3 \\
13.5 \pm 2.9\end{array}$ & 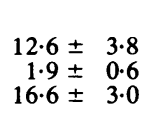 & $\begin{array}{l}23 \cdot 0 \\
26 \cdot 3 \\
19 \cdot 7\end{array}$ & $\begin{array}{l}0.0001 \\
0.0001 \\
0.0001\end{array}$ \\
\hline $\begin{array}{l}\text { WMS Figures } \\
\text { Trial } 1 \text { (\# bits) } \\
\text { Trials to criterion } \\
45 \text { minute delay }\end{array}$ & $\begin{array}{r}13 \cdot 6 \pm 4 \cdot 3 \\
2.1 \pm 1.3 \\
15.0 \pm 3.9\end{array}$ & $\begin{aligned} 17 \cdot 0 \pm & 2 \cdot 8 \\
1 \cdot 0 \pm & 0 \cdot 2 \\
16 \cdot 8 \pm & 2 \cdot 8\end{aligned}$ & $\begin{array}{c}13 \cdot 7 \\
16 \cdot 9 \\
4 \cdot 21\end{array}$ & $\begin{array}{l}0.001 \\
0.0001 \\
0.05\end{array}$ \\
\hline $\begin{array}{l}\text { Brown-Peterson } \\
\text { Total \% correct } \\
\text { No interference } \\
\text { Interference } \\
\text { No delay } \\
4 \text { second delay }\end{array}$ & $\begin{array}{l}59 \cdot 3 \pm 19 \cdot 0 \\
98 \cdot 6 \pm 6 \cdot 4 \\
49 \cdot 6 \pm 23 \cdot 0 \\
45 \cdot 3 \pm 25 \cdot 9 \\
52 \cdot 9 \pm 26 \cdot 3\end{array}$ & $\begin{array}{l}69 \cdot 8 \pm 21 \cdot 1 \\
95 \cdot 2 \pm 8 \cdot 9 \\
63 \cdot 9 \pm 25 \cdot 4 \\
57 \cdot 6 \pm 29 \cdot 7 \\
62 \cdot 1 \pm 28 \cdot 8\end{array}$ & $\begin{array}{l}4 \cdot 77 \\
4 \cdot 19 \\
6 \cdot 06 \\
3 \cdot 37 \\
1 \cdot 91\end{array}$ & $\begin{array}{l}0.04 \\
0.05 \dagger \\
0.02 \\
\text { ns } \\
\text { ns }\end{array}$ \\
\hline
\end{tabular}

*mean \pm SD

†Multiple sclerosis patients better

Table 2 Learning and memory in multiple sclerosis. Predicting test performance with years of active disease, age, acutelquiescent status and education

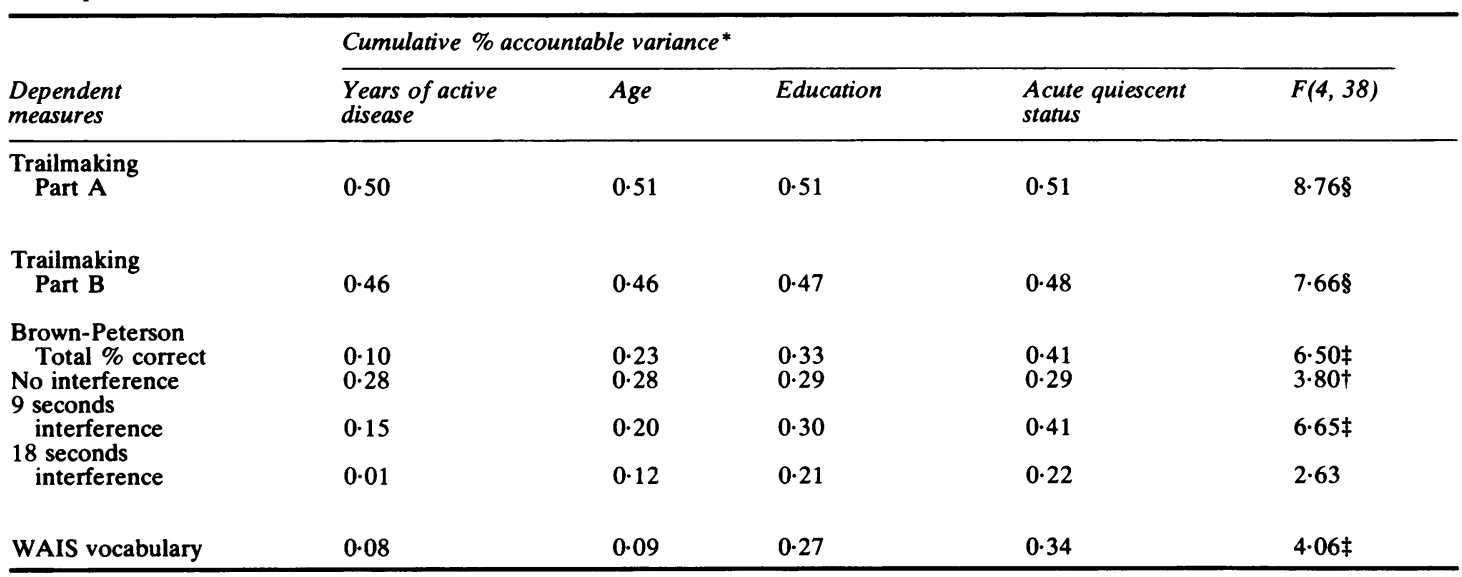

*These are cumulative $R^{2}$ values and can be read as \% variance in test accounted for by addition of each predictor. The $R^{2}$ under column 4 is identical to $R^{2}$ for the entire multiple regression, where $R=$ multiple correlation. Example: For Trails $B$, years of active disease accounts for $46 \%$ of variance in that test; age yields no added predictive power; education adds $1 \%$ prediction, and acute/quiescent status adds a final $1 \%$ for a total $R^{2}=0.48$. The unadjusted multiple $R$ is $\sqrt{ } 0.48$, or 0.69 for this regression.

$\dagger \mathrm{p}<0.05, \neq \mathrm{p}<0.01, \S \mathrm{p}<0.001$.

the same level of difficulty with the cognitively easy Trail Making Part A as with the somewhat more cognitively complex Part B.

The results of the memory tests demonstrated that motor speed and dexterity were not, however, the only influence operating. With the exception of the requirement to reproduce a diagram in the visual section of the Wechsler Memory Scale, none of the other tests had any motor component whatever. Despite this, patients exhibited significant difficulty in short-term retention (Brown-Peterson Test), and, in particular, they appeared to be sensitive to the disrupting effect of enforced mathematical calculations which were introduced as interference. This 


\section{Deficient learning and memory in early and middle phases of multiple sclerosis}

inference is supported by the higher level of statistical separation between performance of patients and nonpatients on the Brown-Peterson "with interference" score than with the "no interference" condition (table 1). Opportunity for rehearsal up to 4 seconds appears to be somewhat, but not dramatically helpful in improving patients' performance.

Turning to learning and delayed recall, it will be seen that patients showed worse immediate recall of both verbal and visual information; they also showed impaired learning in both modalities, since they required more trials to reach the preestablished criterion; finally, although all but two patients did in fact reach the criterion, and many, by virtue of having had more trials received more exposure to the material, patients nevertheless recalled fewer bits of information after 45 min than did their nonpatient counter-parts.

Characteristics of multiple sclerosis patients in respect to neuropsychological functioning

In our efforts to understand potential sources of neuropsychological disadvantage in the patient group, we considered several factors which could theoretically influence performance. These included increasing age, educational disadvantage, length of disease, disease status (that is, flareup vs quiescent), and presence of ACTH or steroid medications. It will be appreciated that many of these putative causal factors are intercorrelated; for example, older patients are likely to have had the disease longer. Similarly, patients who have had the disease longer tend to have more frequent flareups and are more likely to receive steroids or ACTH. For this reason, we decided to explore the possible influence of these various factors on neuropsychological test performance in a multivariate fashion.

In the first set of analyses we performed step-wise multiple regressions to predict various neuropsychological test scores from number of years of active disease, age, acute/quiescent status, and education. Years of active disease was entered first.* Table 2 summarises these regression results. It should be noted that the figures presented are $R^{2}$ values, and therefore can be read as proportion of variance accounted by the variable in question. To derive the actual partial correlation for variables or the multiple correlation it is necessary to take the square root of the values presented. Hence, the multiple correlation for Trails $A$ is the square root of 0.51 , or approximately 0.7 .

\footnotetext{
* Years of active disease is defined as the number of years in which a patient had a minimum of 7 days of significant symptomatology. We constructed this variable rather than the more traditional "years since multiple sclerosis was diagnosed" to avoid artifically equating patients who might have had only a few attacks interspersed with long quiescent periods with patients whose disease was clearly far more active.
}

Turning first to the tests which load on psychomotor speed, it will be seen that the number of years of active disease accounted for most of the variability in Trails $A$ and B. Age, education and active/quiescent status added almost nothing to the explanatory power of these equations. On the other hand, short-term memory (Peterson total) was significantly affected by all four factors-about $10 \%$ of the variance in Peterson errors was explained by each of years that the disease had been active, age, education, and whether an acute flareup had occurred. Further exploration of the Peterson results showed that under the simplest learning conditions (no interference) years of active disease provided the greatest explanatory power; as the complexity of the task increased ( 9 seconds of counting backwards interference) years of active disease, native intelligence (education) and acute/quiescent status all became important. At the most complex level (18 seconds interference) the equation became insignificant. This is explained by the fact that the 18 second condition was so difficult that even nonpatients had problems scoring well on it; indeed, we found no difference between patients and controls on the 18 second interference trial.

Equations which attempted to predict Wechsler memory scores from these four variables failed to reach significance. At the same time, vocabulary score was significantly predicted by education, flareup, and years of active disease.

When we constructed a new set of equations entering medicated/unmedicated status (and dropping out education in order to keep the number of predictor variables to a manageable number) we found that the presence of ACTH or steroids did not provide any additional (unique) explanatory power in the neuropsychological performance of our patients. We believe this to be a reflection of the fact that medication status is very highly correlated with flareup status $\left(\chi^{2}=10 \cdot 1, \mathrm{p}<0.002\right)$.

\section{Discussion}

The results of our study suggest that patients in early and middle phases of multiple sclerosis, beyond exhibiting some of the expected impairments in psychomotor functioning, also suffer decrements in the major domains of memory functioning. These include disturbances in short-term memory, learning, and delayed recall of learned material. It appears that verbal and nonverbal memory are affected equally. Efficiency of short-term retention seems to be particularly vulnerable to the effects of proactive interference, and the opportunity to rehearse for up to 4 seconds does not appear to be substantially helpful in correcting this difficulty with 
short-term retention. It appears further that the defect in short-term storage of information is related to the number of years that a patient's disease has been active, and to the activity of the disease (flareup vs quiescence) at the time of testing. Medication with ACTH or steroids, on the other hand, does not appear to explain short-term retention deficit; but this might be due to our difficulty in disentangling the possible independent effects of acute flareup and drugs prescribed for that flareup.

How do our findings relate to previous studies of memory functioning in multiple sclerosis? As we indicated in our introductory remarks, very few neuropsychological studies of this disorder performed systematic explorations of memory. Surridge $^{13}$ performed ratings of memory on his 108 patients on the basis of a clinical psychiatric examination. He reported that approximately two-thirds of his patients had some intellectual deterioration, and that the typical difficulty was amnesia. The companion study by Jambor ${ }^{14}$ examined most of these same patients with several formal memory tests. Jambor found that multiple sclerosis patients had difficulties with a sentence learning task, requiring more trials than controls to learn the Babcock Sentences. This result is comparable to our present observation that patients with multiple sclerosis required more trials to learn our prose passage. Jambor also reported that his patients had difficulties on a task involving delayed recall of pictures. This appears similar to our observation that delayed recall of figures is impaired in multiple sclerosis, but the lack of detail in Jambor's report makes direct comparison of our results problematic. In relating these Surridge and Jambor data to our own, it is important to recognise that their patients were, in general, ill for substantially longer periods than ours. For example, approximately half of their patients were ill for 6 to 10 years, and another third had the disease for 11 to 25 years. In contrast, $60 \%$ of our patients were within the first five years of their initial diagnosis, $30 \%$ were 6 to 10 years post diagnosis, and only $10 \%$ were 11 to 15 years post diagnosis. The fact that we found our sample to have memory difficulties which were qualitatively similar to those of Surridge and Jambor suggests that memory disturbance might begin evolving relatively early in the natural history of multiple sclerosis.

The second systematic study of memory in multiple sclerosis was that of Beatty and Gange ${ }^{15}$ whose 26 patients had a mean disease length of 9 years. The task they employed, which involved free recall of 24 words presented at 2 second intervals for four trials, is not directly comparable to our procedure, though it probably has most in common with our story learning to criterion technique. These authors found that their patients recalled significantly fewer words over the four trials than did their controls, and that although the patients benefited somewhat from repeated testing, they did not gain at the same rate as the nonpatients. Beatty and Gange's multiple sclerosis patients were also deficient on a verbal delayed recall task, which consisted of 25 multiple choice questions about a short story presented 30 min previously. This finding is consistent with our observation that patients had difficulty recalling a prose paragraph after $45 \mathrm{~min}$

The third neuropsychological study to assess learning and memory was that of Staples and Lincoln. ${ }^{16}$ Their approach to memory assessment was more comprehensive than that of the other two studies, involving as it did the full Wechsler Memory Scale ${ }^{18}$ with the addition of verbal and visual delayed recall requirements. Part of their study involved comparing performance of multiple sclerosis patients to published norms, while another part compared 29 patients with multiple sclerosis to a matched group of muscular dystrophy patients. The results of the controlled investigation showed that patients with multiple sclerosis were impaired in immediate recall of Wechsler figures but not stories; that they were impaired on delayed recall both of verbal and nonverbal material; and that their verbal-verbal paired associate learning was also deficient. Once again, we would note that the patients in the Staples and Lincoln study were described by the authors as having "severe longstanding disability" with a mean duration of 15.9 years.

We consider that our findings both confirm and extend the results of these several previous investigations into the nature of memory disturbance in multiple sclerosis. Our study is distinctive in two respects. It is the first of which we are aware that has focused on processes of short-term memory, which are presumed to reflect the initial encoding of information. Although the neuroanatomic underpinnings of memory are still imperfectly understood, it is thought that the lesions of the dorsomedial thalamic nuclei are especially likely to be associated with deficits in learning, whereas medial temporal lobe pathology interferes with ability to recall information which was once learned. In the latter instance, there appears to be a lateralisation of function such that recall of verbal material is influenced by the left temporal lobe, while recall of nonverbal material is related to right temporal function. ${ }^{24}$ If these neuroanatomic models are correct, then our findings would suggest that many of our patients have foci of demyelination in the diencephalon while others may have lesions bilaterally in the hippocampus and related temporal structures. Unfor- 
tunately, brain imaging (CT scanning) was not sufficiently sophisticated in our study to detect small lesions in these areas. With the advent of nuclear magnetic resonance (NMR) technology it will soon become feasible to validate these neuropsychological observations of memory disturbance with detailed information concerning the distribution, size, and activity of plaques of demyelination.

The second unique feature of our study has to do with the phase in the natural history of their disease in which our patients found themselves. The majority experienced active disease for less than 5 years, and there were only four patients whose disease had been active 11-15 years. Further, only two of our patients were chronically hospitalised, the rest living either independently or with family in the community. Despite their relative skew toward lack of gross physical or cognitive disability, our patients did manifest substantial memory difficulties.

In summary, patients in early and middle phases of multiple sclerosis often showed disturbances in short-term memory, learning, and recall of verbal and nonverbal information. Some of these memory disturbances were correlated with number of years of active disease, acute versus quiescent status of the disorder, age, and background educational level of the patient. ACTH and steroid medications appeared to play little or no role in the memory difficulties. We conclude that subtle, unrecognised memory disturbance is common even in early multiple sclerosis and recommend that future, more detailed investigations of memory and other neuropsychological functions be coupled with advanced brain imaging technology.

The authors are indebted to Dr PC Gautier-Smith, for his enthusiastic support of this study, and particularly, his willingness to identify patients who might be suitable as research subjects. Professors John Marshall, Newsom-Davis, and PK Thomas were also helpful in allowing us access to patients, as were Drs RW Ross Russell, JA Morgan-Hughes, $P$ Rudge, AN Gale, R Clifford Jones, AJ Lees, and E Byrne. Ms Marge Zeitsman assisted in scheduling some of our patients, and Ms Debi Taylor helped in the preparation of this manuscript.

This research was conducted while Dr I Grant was honorary research fellow at the Institute of Neurology, supported by the Foundations Fund for Research in Psychiatry, 1980-81.

\section{References}

' Trimble MR, Grant I. Psychiatric aspects of multiple sclerosis. In: Benson DF, Blumer D, eds. Psychiatric
Aspects of Neurologic Disease, Volume II. New York: Grune and Stratton, 1982:279-99.

2 Marsh G. Disability and intellectual function in multiple sclerosis. J Nerv Ment Dis 1980;168:758-62.

${ }^{3}$ Ross AT, Reitan RM. Intellectual and affective functions in multiple sclerosis. Arch Neurol 1955;73:663-77.

${ }^{4}$ Parsons OA, Stewart BA, Arenberg D. Impairment of abstracting ability in multiple sclerosis. J Nerv Ment Dis 1957;125:221-5.

${ }^{5}$ Matthews CG, Cleeland CS, Hopper CL. Neuropsychological patterns in multiple sclerosis. Dis Nerv Syst 1970;31:161-70.

- Reitan RM, Reed JC, Dyken M. Cognitive, psychomotor, and motor correlates of multiple sclerosis. J Nerv Ment Dis 1971;153:218-24.

${ }^{7}$ Goldstein G, Shelly CH. Neuropsychological diagnosis of multiple sclerosis in a neuropsychiatric setting. $J$ Nerv Ment Dis 1974;158:280-90.

${ }^{8}$ Peyser JM, Edwards KR, Poser CM, Filskov SB. Cognitive function in patients with multiple sclerosis. Arch Neurol 1980;37:577-9.

${ }^{9}$ Ivnik RJ. Neuropsychological stability in multiple sclerosis. J Consult Clin Psychol 1978;46:913-23.

${ }^{10}$ Ivnik RJ. Neuropsychological test performance as a function of duration of multiple sclerosis-related symptomatology. J Clin Psychol 1978;39:304-7.

${ }^{1}$ Charcot JM. Lectures on the Diseases of the Nervous System Delivered at La Saltpetriere (trans G. Sigerson). London: The New Sydenham Society, 1877:194-5.

${ }^{12}$ Gowers, WR. A Manual of Diseases of the Nervous System, 2nd Ed., London: Churchill, 1893.

${ }^{13}$ Surridge D. An investigation of some psychiatric aspects of multiple sclerosis. Br J Psychiatry 1969;155:74964.

${ }^{14} \mathrm{Jambor} \mathrm{KL}$. Cognitive functioning in multiple sclerosis. Br J Psychiatry 1969;115:765-75.

${ }^{15}$ Beatty PA, Gange JJ. Neuropsychological aspects of multiple sclerosis. J Nerv Ment Dis 1977;164:42-50.

${ }^{16}$ Staples D, Lincoln NB. Intellectual impairment in multiple sclerosis. Rheumatol Rehab 1979;18:153-60.

17 Poser CM, Paty DW, Scheinberg L, et al. New diagnostic criteria for multiple sclerosis: Guidelines for research protocols. Ann Neurol 1983;13:227-31.

${ }^{18}$ Wechsler D. Wechsler Adult Intelligence Scale. New York: The Psychological Corporation, 1955.

19 Reitan RM. Validity of the Trail Test as an indicator of organic brain damage. Percept Mot Skills 1958;8:271-6.

${ }^{20}$ Wechsler D, Stone C. Wechsler Memory Scale. New York: The Psychological Corporation, 1945.

${ }^{21}$ Baddeley AD. The Psychology of Memory. New York: Basic Books, 1976.

${ }^{22}$ Peterson LR, Peterson MJ. Short-term retention of individual verbal items. J Exp Psychol 1959;58:193-8.

${ }^{23}$ Butters N, Sax D, Montgomery K, Tarlow S. Comparison of the neuropsychological deficits associated with early and advanced Huntington's Disease. Arch Neurol 1978;35:585-9.

${ }^{24}$ Squire LR. The neuropsychology of human memory. Ann Rev Neurosc 1982;5:241-73. 